\title{
Correction Factor for Power Gain in Antenna Arrays
}

\author{
Vinay B. Ramakrishnaiah, Robert F. Kubichek, Suresh S. Muknahallipatna \\ Department of Electrical and Computer Engineering, University of Wyoming, Laramie, WY, USA \\ Email: sureshm@uwyo.edu
}

How to cite this paper: Ramakrishnaiah, V.B., Kubichek, R.F. and Muknahallipatna, S.S. (2018) Correction Factor for Power Gain in Antenna Arrays. Int. J. Communications, Network and System Sciences, 11, 45-52.

https://doi.org/10.4236/ijcns.2018.113004

Received: February 1, 2018

Accepted: March 25, 2018

Published: March 28, 2018

Copyright $\odot 2018$ by authors and Scientific Research Publishing Inc. This work is licensed under the Creative Commons Attribution International License (CC BY 4.0).

http://creativecommons.org/licenses/by/4.0/

\begin{abstract}
Antenna array gain is a relative measure of performance defined differently in various literature. Most definitions of gain are not power consistent, and thus cannot be used directly in link budget analysis. In this short paper, we present a power correction factor for common definitions of power gain of antenna arrays that allows them to be used in standard link budget calculations.
\end{abstract}

\section{Keywords}

Antenna Arrays, Power Gain, Gain Correction, Link Budget

\section{Introduction}

A radio antenna is a transducer that converts electric currents into radio waves and vice versa. Antenna geometry determines the directional characteristics of each antenna. Combining several antenna elements into an array can yield higher gain and interference suppression. The overall radiation pattern determines directivity of the antenna array and the gain of the array is defined as the product of directivity and the antenna array efficiency. The antenna array gain is defined as the ratio of the intensity in a given direction to the radiation intensity that would be obtained if the same power is radiated using a single isotropic radiator. The resulting voltage gain is called the array factor, and it is a frequent practice to use the square of the array factor to indicate the power gain of the antenna array. In this paper, we show that this widespread practice results in inaccurate power values, and we present a correction factor to make the power gain directly applicable to link budget calculations.

If a signal $s(t)$ is transmitted, the signal received by an antenna array is represented as $y(t)$. For example, the received signal in case of a uniform linear array (ULA) is 


$$
\boldsymbol{y}(t)=\left[\begin{array}{c}
1 \\
\mathrm{e}^{j k d \cos \theta} \\
\vdots \\
\mathrm{e}^{j(M-1) k d \cos \theta}
\end{array}\right] s(t)+\left[\begin{array}{c}
v_{0}(t) \\
\vdots \\
v_{M-1}(t)
\end{array}\right]
$$

or $\boldsymbol{y}(t)=\boldsymbol{h}(\theta) s(t)+\boldsymbol{V}$, where $k, d$, and $\theta$ are the wavenumber, antenna element separation, and direction of arrival (DOA), respectively, and $\boldsymbol{h}(\theta)$ is called the steering vector. Note that $\boldsymbol{h}(\theta)$ must be modified for each specific antenna array geometry to give proper delay characteristics in the direction $\theta$.

It is possible to find a filter, $w$, that amplifies the signals in certain directions,

$$
\boldsymbol{y}(t)=\boldsymbol{w}^{*} \boldsymbol{h}(\theta) s(t)+\boldsymbol{w}^{*} \boldsymbol{V},
$$

where $(.)^{*}$ indicates the complex conjugate transpose. The term $\boldsymbol{w}^{*} \boldsymbol{h}(\theta)$, also known as the array factor, represents the voltage gain of an antenna array, and is a function of $\theta$ (assuming 2D patterns). A lot of literature considers the antenna array gain to be equal to either the array factor $(\mathrm{AF})$ or the power gain,

$$
G_{p}(\theta, \phi)=|A F(\theta, \phi)|^{2} G(\theta, \phi)
$$

where $G(\theta, \phi)$ is the power gain of a single element of the array, and $\phi$ represents the other Euler angle for 3D coordinates.

Many papers [1] [2] [3] [4] [5] analyze antenna array characteristics using the gain parameter $G_{p}(\theta, \phi)$. For example, the authors of [5] study the impact of array element spacing and the number of elements on the directivity of the antenna array. They report the AF as the gain of the antenna array and show that the half-power beam width and the number of side lobes increase with increasing element spacing. However, as we show in this paper, such analysis can give misleading results unless $G_{p}(\theta, \phi)$ is properly normalized to preserve the actual power amplification provided by the antenna.

While the array factor provides the complex amplitude gain for an array, it does not directly relate to the power gain. As an example, consider a passive two-element transmitter antenna array with weight vector $w=[1,1]^{\mathrm{T}}$. The AF of this antenna array in one coordinate angle $\theta$ (for simplicity) is equal to $1+\mathrm{e}^{j k d \cos \theta}$. Assuming unit elemental power gain, i.e., $G(\theta)=1$, the total power gain,

$$
G_{\text {tot }}=\frac{1}{2 \pi} \int_{0}^{2 \pi}\left|1+\mathrm{e}^{j k d \cos \theta}\right|^{2} \mathrm{~d} \theta=2\left(1+J_{0}(2 d k)\right),
$$

where $J_{0}$ is the Bessel function of the first kind. Passive antenna arrays should yield $G_{\text {tot }} \leq 1$, where equality holds for purely lossless systems. However, the expected result is not obtained; for example, using a quarter wavelength antenna separation, the result is $G_{t o t}=1.47$. Clearly, the typical approach of using the squared array factor will not yield accurate results for applications such as link budget analysis unless an appropriate scaling factor is used.

Literature like [6] [7] uses normalized AF in their work. They consider an $M$ element uniform linear array with unit weights, such that the 


$$
A F=w^{*} h=\left[1+\mathrm{e}^{j k d \cos \theta}+\cdots+\mathrm{e}^{j(M-1) k d \cos \theta}\right] .
$$

Defining $k d \cos \theta \triangleq \psi$,

$$
A F \mathrm{e}^{j \psi}=\mathrm{e}^{j \psi}+\mathrm{e}^{j 2 \psi}+\cdots+\mathrm{e}^{j(M-1) \psi} .
$$

Subtracting (1) from (2) and using the Taylor series definition of the sine function, the array factor becomes

$$
A F=\mathrm{e}^{j(M-1) \frac{\psi}{2}}\left(\frac{\sin \frac{M \psi}{2}}{\sin \frac{\psi}{2}}\right)
$$

The unnormalized power gain of the antenna array is

$$
g=|A F|^{2}=\left|\frac{\sin \frac{M \psi}{2}}{\sin \frac{\psi}{2}}\right|^{2}
$$

and the gain is normalized to have unity gain at DC, which is at zero wavenumber $\psi=0$, or $\theta=90^{\circ}$. Therefore, the authors consider the normalized power gain of the antenna array to be

$$
g=\left|\frac{\sin \frac{M \psi}{2}}{M \sin \frac{\psi}{2}}\right|^{2}
$$

Again, considering the example of a 2-element ULA with quarter wavelength spacing with this normalization factor, the integral of the gain over all look angles is,

$$
\frac{1}{2 \pi} \int_{0}^{2 \pi}\left|\frac{\sin \frac{\pi}{2} \cos \theta}{2 \sin \frac{\pi}{4} \cos \theta}\right|^{2} \mathrm{~d} \theta=\frac{1}{2}\left(1+J_{0}\left(\frac{\pi}{2}\right)\right) \approx 0.74
$$

This shows that the method of normalization presented in [6] [7] does not represent the actual power gain of the antenna array as the equation does not integrate to 1 even for a lossless system.

\section{Power Gain of Antenna Arrays}

By inspection, the scaling factor that normalizes the integral of the square of the AF over all look angles must be

$$
\rho=\frac{1}{2 \pi} \int_{0}^{2 \pi}|A F(\theta)|^{2} \mathrm{~d} \theta
$$

ensuring total power in the system is conserved. Therefore, the power gain of a lossless passive antenna array is

$$
G_{p}(\theta)=\frac{1}{\rho}|A F(\theta)|^{2}=2 \pi \frac{|A F(\theta)|^{2}}{\int_{0}^{2 \pi}|A F(\theta)|^{2} \mathrm{~d} \theta} .
$$


Analytically, the problem can also be approached as follows. The directive gain or directivity of a passive antenna is defined as the ratio of the radiation intensity in a given direction from the antenna to the radiation intensity averaged over all directions [8] and the power gain of the antenna, $G_{p}(\theta, \phi)$ is related to the directivity, $D(\theta, \phi)$ as $G_{p}(\theta, \phi)=\eta D(\theta, \phi)$, where $\eta$ is the antenna radiation efficiency. For an antenna that is $100 \%$ efficient, the directivity is equal to the gain of the antenna. Using the definition of directivity, it can be written in mathematical form as

$$
D=\frac{U}{U_{0}}=\frac{4 \pi U}{P_{\text {rad }}},
$$

where $U$ is the radiant intensity, $U_{0}$ is the radiant intensity of isotropic source, and $P_{r a d}$ is the total radiated power. The radiant intensity of the antenna [6] [7] is of the form

$$
U=B_{0} F(\theta, \phi) \simeq \frac{1}{2 \eta}\left[\left|E_{\theta}^{0}(\theta, \phi)\right|^{2}+\left|E_{\phi}^{0}(\theta, \phi)\right|^{2}\right]
$$

where $B_{0}$ is a constant, $F$ is the normalized radiation intensity, and $E^{0}$ represents the antenna's far-zone electric field components. The total radiated power is found using the surface integral $P_{\text {rad }}=\oiint U(\theta, \phi) \mathrm{d} \Omega=B_{0} \int_{0}^{2 \pi} \int_{0}^{\pi} F(\theta, \phi) \sin \theta \mathrm{d} \theta \mathrm{d} \phi$. Therefore, the general expression for directivity is given by

$$
D(\theta, \phi)=4 \pi \frac{F(\theta, \phi)}{\int_{0}^{2 \pi} \int_{0}^{\pi} F(\theta, \phi) \sin \theta \mathrm{d} \theta \mathrm{d} \phi} .
$$

The normalized radiant intensity is proportional to the square of the array factor, i.e., $F(\theta, \phi)=\alpha|A F|^{2}$. Using this relation and representing Equation (8) in only one coordinate angle $\theta$, the gain of an antenna that is $100 \%$ efficient is given by

$$
G_{p}(\theta)=2 \pi \frac{|A F(\theta)|^{2}}{\int_{0}^{2 \pi}|A F(\theta)|^{2} \mathrm{~d} \theta},
$$

which is exactly same as Equation (7) that was obtained using the law of conservation of energy.

In order to compute the power gain of an active antenna, consider the simplified representation of the transmitter array shown in Figure 1.

We assume that the system consists of a signal generator with power, $P=v i$, active amplifiers, and passive phase shifters to realize the antenna weights. Assuming the power from the signal generator is split equally across all the antennas in the array, the current is equally divided into $i_{j}=i / M$ in each antenna branch, whereas the voltage remains the same. Assuming an ideal voltage amplifier, the power required by each amplifier to realize the active weights is $\frac{\left|w_{j}\right| v i}{M}-\frac{v i}{M}=\frac{v i}{M}\left(\left|w_{j}\right|-1\right)$. Therefore, the total power injected into the antenna system is the sum of the power generated by the signal generator and the power 


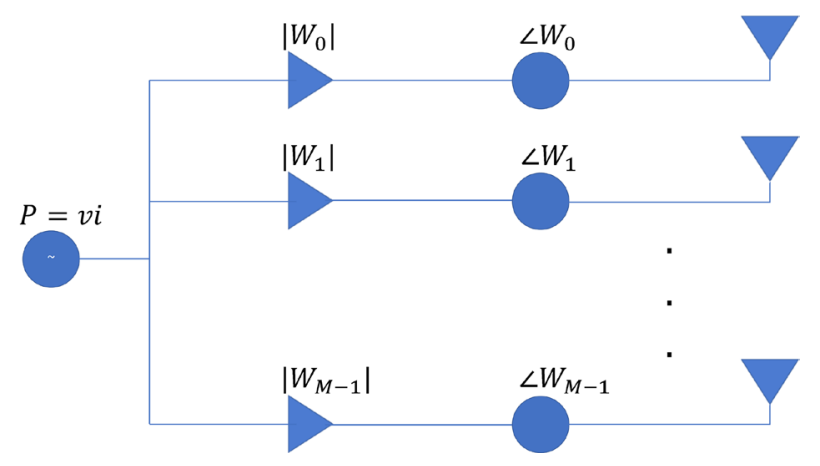

Figure 1. Simplified antenna array system.

required by the amplifiers. Mathematically, $P_{t}=v i+\frac{v i}{M} \sum_{0}^{M-1}\left(\left|w_{j}\right|-1\right)$, which simplifies to the expression,

$$
P_{t}=\frac{v i}{M} \sum_{j=0}^{M-1}\left|w_{j}\right| .
$$

Considering the example of a passive antenna array using phase-only weights, i.e., $\left|w_{j}\right|=1, \forall j$, the total power injected into the antenna system computed using Equation (10) is $P_{t}=v i$. Equation (10) indicates that the antenna array amplifies the transmitter power, $P$ by a factor of

$$
P_{\text {fact }}=\frac{1}{M} \sum_{j=0}^{M-1}\left|w_{j}\right|,
$$

which simplifies to $P_{\text {fact }}=1$ for a lossless passive array. Therefore, the power gain of an active antenna array is given by

$$
G_{p a}(\theta)=2 \pi \frac{|A F(\theta)|^{2}}{\int_{0}^{2 \pi}|A F(\theta)|^{2} \mathrm{~d} \theta} P_{\text {fact }} .
$$

\section{Comparison of the Lossless Passive and the Minimum Variance Distortion-Less Response Array}

A lossless passive array antenna is a phased array with magnitude of the antenna weights equal to the 1. On the other hand, considering Equation (1), it is possible to find the antenna weights, $w$ (a linear filter) that minimize the effects of noise without distorting the signal:

$$
\begin{gathered}
\hat{s}=\sum w_{i} y_{i}=\boldsymbol{w}^{*} \boldsymbol{y} . \\
E[\hat{s}]=E\left[\boldsymbol{w}^{*} \boldsymbol{y}\right]=E\left[\boldsymbol{w}^{*} \boldsymbol{h}(\theta) s+\boldsymbol{w}^{*} \boldsymbol{V}\right]=\boldsymbol{w}^{*} \boldsymbol{h}(\theta) s=s .
\end{gathered}
$$

$\boldsymbol{w}^{*} \boldsymbol{h}\left(\theta_{s}\right)=1$ gives unit gain in the direction of interest, $\theta_{s}$. The error is given by

$$
\tilde{s}=s-\hat{s}=s-\boldsymbol{w}^{*} \boldsymbol{h}(\theta) s-\boldsymbol{w}^{*} \boldsymbol{V}=\boldsymbol{w}^{*} \boldsymbol{V},
$$

and the mean squared error is

$$
M S E=E\left[\boldsymbol{w}^{*} \boldsymbol{V} \boldsymbol{V}^{*} \boldsymbol{w}\right]=\boldsymbol{w}^{*} \boldsymbol{R}_{v} \boldsymbol{w},
$$


where $\boldsymbol{R}_{v}$ is the autocorrelation matrix. Applying the Gauss-Markov theorem to get the minimum mean squared error, we obtain

$$
\boldsymbol{w}^{*}=\frac{\boldsymbol{h}^{*}(\theta) \boldsymbol{R}_{v}^{-1}}{\boldsymbol{h}^{*}(\theta) \boldsymbol{R}_{v}^{-1} \boldsymbol{h}(\theta)} .
$$

This is called a "Minimum Variance Distortion-less Response (MVDR) [9]" filter.

MVDR weights produce a unit gain in the direction of interest similar to the array response as shown in Equation (5). Figure 2 shows the array factor of an MVDR array at a desired direction of $\frac{\pi}{2}$, compared to a passive array with unit weights for uniform linear geometry of 100 antennas. Here, the noise covariance matrix is assumed to be the identity matrix. It can be seen from Figure 2 that the MVDR response is a scaled version of the passive array response, scaled down to have a maximum gain of unity. This makes the MVDR array a lossy system, which could be realized using step attenuators in hardware. We next compare the lossless passive antenna array with the lossy MVDR array to re-iterate the fact that the typical definition of power gain provides inaccurate values if not corrected for power, and this cannot be directly used for link budget calculations.

Using the passive and MVDR weights, Matlab simulations were conducted to observe the variation of the overall power output from an antenna array with increasing number of antennas (M), and the results are shown in Figure 3. The scaling factor, $\rho$ is the integral of the square of the array factor as shown in Equation (6), which gives the total power gain in all directions. The scaling factor for a lossy system (MVDR) must be less than 1, whereas, the scaling factor for a lossless passive array must be equal to 1 as total power output from the array is equal to the total power input. It is clear from Figure 3 that by using the typical definition of the power gain, the overall power output (scaling factor) for a passive array is greater than 1 and it varies with the number of antennas in the array. This shows that the common definition of the power gain, as the square of the array factor, is not power accurate and must be used with caution.
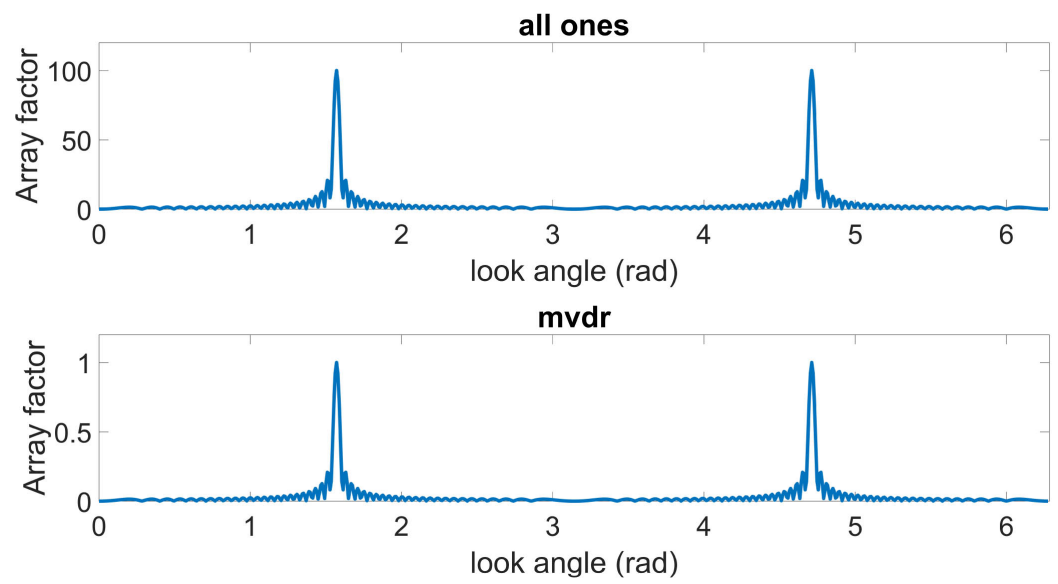

Figure 2. Antenna array response for a passive array compared to MVDR weights for a uniform linear array of 100 antennas. 


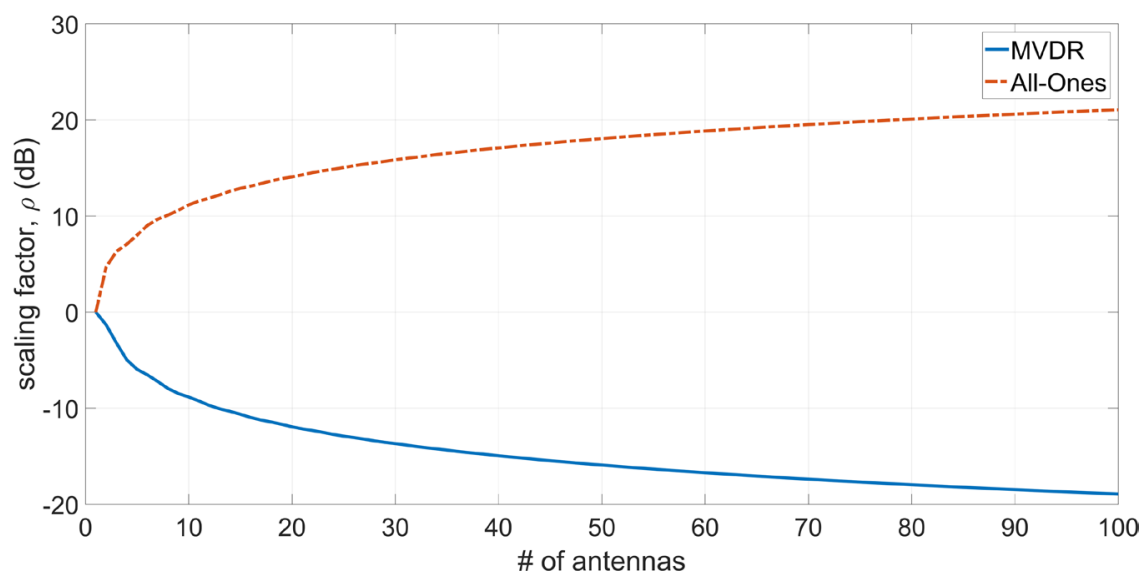

Figure 3. The variation of the scaling factor, $\rho$, for passive array and MVDR array with varying number of antennas.

As an example, consider a link budget calculation with the following parameters: Transmitter power, $P_{t}=10 \mathrm{~W}$, receiving antenna gain, $G_{r}=1$, and the distance between the transmitter and receiver, $d=1 \mathrm{~km}$. With the assumption of free-space propagation and operating frequency of $900 \mathrm{MHz}$, the path loss, $L=\left(\frac{4 \pi d}{\lambda}\right)^{2}=\left(12 \pi \times 10^{3}\right)^{2}$. Using a uniform linear array with 10 antennas (with maximum amplitude gain of 10) and passive weights (all ones), the received power in the direction of maximum gain $\left(\theta=\frac{\pi}{2}\right)$ by means of the common definition of power gain as the square of the array factor is

$$
\begin{gathered}
P_{r_{\text {uncorrected }}}(\mathrm{dB})=P_{t}(\mathrm{~dB})+G_{t_{\text {uncorrected }}}(\mathrm{dB})+G_{r}(\mathrm{~dB})-L(\mathrm{~dB}) \\
P_{r}=10 \log _{10} 10+20 \log _{10} 10+10 \log _{10} 1-20 \log _{10}\left(12 \pi \times 10^{3}\right)=-61.5266 \mathrm{dBW} .
\end{gathered}
$$

Using the graph in Figure 3 and applying the correction factor for a lossless passive array with 10 antennas, the transmitting antenna gain is $G_{t_{\text {corrected }}}=G_{t_{\text {tucorrected }}}-\rho+P_{\text {fact }}=20 \log _{10} 10-11.1529+0=8.8471 \mathrm{dBW}$. Therefore, the received power is equal to

$$
P_{r_{\text {corrected }}}=10 \log _{10} 10+8.8471+10 \log _{10} 1-20 \log _{10}\left(12 \pi \times 10^{3}\right)=-72.6795 \mathrm{dBW} \text {, }
$$

which is less than the uncorrected power, $P_{r_{\text {uncoreceted }}}$.

\section{Conclusions}

Antenna array gain is a relative measure of performance and can be defined in several ways. Some literature considers the magnitude square of the array factor (AF) as the power gain while others normalize the square of the AF to have a unit D.C. gain. The normalized array gain makes it easier for comparing different array patterns by having them on a same scale, whereas the magnitude square of the AF can be directly analyzed to study the effects of changing array geometry. Unfortunately, these methods are not suitable indicators of the antenna array power gain. 
Typically, the antenna gain is indicated in $\mathrm{dBi}$, i.e., providing the gain of an array as a ratio with respect to the gain of an isotropic radiator. However, most literature does not discuss gain corrected for power. In this paper, we present an approach of using a correction factor to correct for power, so that the integral of the power gain for a lossless passive antenna equals unity. Later, we also provide a power scaling factor to account for active array antennas. The discussed numerical and simulated examples of computing the passive and MVDR weights show how the overall power gain varies with the number of antennas if not corrected for power. The discussed method of applying the correction factor should be directly applicable to link budget calculations.

\section{References}

[1] Bhamidipati, S.Y.S. (2012) Low Complexity Hybrid Digital/Analog Phased Array for Ad Hoc Networks. University of Wyoming, Laramie.

[2] Boyd, S. and Vandenberghe, L. (2004) Convex Optimization. Cambridge University Press, Cambridge. https://doi.org/10.1017/CBO9780511804441

[3] Sadler, D.J. (2005) Sector Beam Synthesis Using Antenna Arrays. The IEE Signal Processing Professional Network and EURASIP, 29. https://doi.org/10.1049/ic:20050399

[4] Orfanidis, S.J. (2002) Electromagnetic Waves and Antennas. Rutgers University, New Brunswick, NJ.

[5] Maharimi, S.F., Malek, M.F.A., Jamlos, M.F., Neoh, S.C. and Jusoh, M. (2012) Impact of Number Elements on Array Factor in Linear Arrays Antenna. IEEE 8 th In ternational Colloquium on Signal Processing and Its Applications (CSPA, Melaka, 23-25 March 2012.

[6] Haupt, R.L. (2010) Antenna Arrays: A Computational Approach. John Wiley \& Sons, Inc., Hoboken, New Jersey. https://doi.org/10.1002/9780470937464

[7] Balanis, C.A. (2005) Antenna Theory: Analysis and Design. John Wiley \& Sons, Inc., Hoboken, New Jersey.

[8] (2013) 145-2013-IEEE Standard for Definitions of Terms for Antennas.

[9] Kay, S.M. (2013) Fundamentals of Statistical Signal Processing: Practical Algorithm Development. Vol. 3, Pearson Education, Westford, Massachusetts. 